\title{
TRANSPLANTATION OF BONE MARROW DERIVED PROGENITOR CELLS IN ACUTE MYOCARDIAL INFARCTION. THE FIRST RESULTS
}

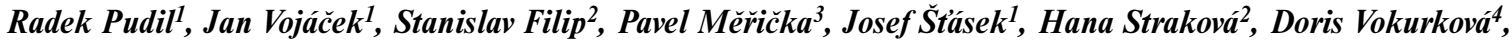 \\ Miroslav Solar̆ $\check{r}^{1}$ Jaroslav Mokrý, Jan Dominik ${ }^{6}$, Jaroslav Malý
}

\begin{abstract}
Charles University Prague, Faculty of Medicine in Hradec Králové and University Hospital Hradec Králové, Czech Republic: 1st Department of Medicine ${ }^{1}$, Department of Oncology and Radiology ${ }^{2}$, Tissue Bank ${ }^{3}$, Department of Clinical Immunology and Allergology ${ }^{4}$, Department of Histology and Embryology ${ }^{5}$, Department of Cardiosurgery ${ }^{6}$, 2nd Department of Medicine ${ }^{7}$
\end{abstract}

\begin{abstract}
Summary: The intracoronary administration of autologous bone marrow cells (BMCs) has been shown to improve the left ventricle function in the course of acute myocardial infarction.

Therefore we have started a clinical trial using transplantation of BMCs in the acute phase of myocardial infarction. The aim of our study is to assess the feasibility and safety of this procedure, and effect on the left ventricle function of these patients. We describe the first experience in two patients with acute myocardial infarction reperfused using direct stenting. The aspiration of bone marrow from the sternum provided sufficient amount of the cells for transplantation. No serious ischemia and no changes in coronary artery patency were detected after intracoronary infusion. The left ventricle ejection fraction was increasing throughout the time of three-month follow-up. No other complications (ventricular arrhythmias, reinfarction, thrombus formation) were detected.
\end{abstract}

Key words: Myocardial infarction; Bone marrow cells; Progenitor cells; Ejection fraction

\section{Introduction}

The first results of the clinical studies using the intracoronary administration of bone marrow progenitor cells (BMC) after successful revascularization in the course of acute myocardial infarction (MI) are promising (2). It has been reported that improvement of the left ventricle function is caused by the stimulation of neovascularization and the enhancement of cardiac myocyte regeneration in the infarcted area $(1,2,3,4,5,6)$. The previous observations and clinical studies have shown that this method is feasible and safe (9).

The aim of our study is to investigate the feasibility, safety, and initial clinical outcome of intracoronary infusion of autologous progenitor cells in patients with acute MI. The present report describes our first experience with this method.

\section{Material and Methods}

\section{Patients}

Two patients over 18 years with ST-segment elevation extensive acute MI treated with direct stenting are included into the study. Exclusion criteria are the presence of cardiogenic shock (defined as the presence of hypotension systolic blood pressure $<80 \mathrm{mmHg}$ requiring catecholamine treatment or intraaortic balloon counterpulsation), women in reproductive age, haematologic diseases, major bleeding requiring blood substitution, serious liver and kidney diseases, any malignant disease in the past or at present, immunodeficiency, viral or bacterial infection, or unwillingness to participate. The study protocol was approved by The Ethics Committee of the University Hospital in Hradec Králové, and the study was conducted in accordance with the Declaration of Helsinky. Written informed consent was obtained from both patients.

\section{Preparation and characterization of transplanted cells, transplantation protocol}

Fifty $\mathrm{mL}$ of bone marrow aspirate was obtained from the sternum into a sterile plastic container with $7 \mathrm{ml}$ of ACD-A solution (Baxter SA, Belgium). Nucleated bonemarrow cells were isolated by density gradient sedimentation in 6\% dextran with ACD-A solution (ratio 6:1) followed by centrifugation of isolated cells in the same solution. The final cell concentration was adjusted by diluting with dex- 
tran ACD solution (mentioned above) and divided into 3 syringes each containing $3 \mathrm{~mL}$ of suspension of bone marrow nucleated cells. Cell manipulation was made in a clean room with validated grade $\mathrm{A}$ with the background $\mathrm{B}$. The cell suspension consisted of heterogenous cell populations including hematopoietic progenitor cells and other cell types (eg. side population cells, stroma cells, etc.). Hematopoietic progenitor cells were determined by flow-cytometry analyses using directly monoclonal antibodies against antihuman CD 34 (QBend PE, Immunotech Marseille, France) and CD 133 (QBend PE, Miltenyi Biotec Gmbh, Germany) as well as by culture of CFU-GM and BFU-E. Mesenchymal cells were cultured as well. After ex-vivo preparation, the aspirate was transplanted within 24 hours. Cells were infused via an over-the-wire balloon catheter advanced into the stent previously implanted during the acute reperfusion procedure. The amount of $3 \mathrm{~mL}$ of progenitor cell suspension was infused during low-pressure balloon inflation with the aim to completely block blood flow for 2-3 minutes and to allow for adhesion and transmigration of the infused cells through the endothelium. The mononucleated cell doses were $3,72 \times 10^{7}$ and $2,35 \times 10^{7}$, number of CFU-GM was $2,34 \times 10^{5}$ and $1,29 \times 10^{5}$, respectively.

This maneuver was repeated three times to accommodate infusion of the total 12 and $15 \mathrm{~mL}$ respectively progenitor cell suspension, interrupted by 3 minutes of reflow by deflation of the balloon to minimize the extension of ischemia. After completion of intracoronary cell transplantation, coronary angiography was repeated to ascertain vessel patency and unimpeded flow of contrast material.

Before and after procedure, plasma levels of creatine-kinase, $\mathrm{MB}$, and troponin $\mathrm{T}$ were assessed with the aim to analyse the presence of serious myocardial ischemia.

The function and regional motion defects of the left ventricle were assessed using echocardiography which was performed before, 1 week, one, and three months after the procedure.

\section{Preliminary results}

The study started in October 2004 when the first two patients were treated using the progenitor cell transplantation. Both patients (males, aged 37 and 54 years, first manifestation of coronary artery disease, no positive family history) were admitted to hospital for ST-segment elevation anteroseptal MI. Direct PCI (percutaneous coronary intervention) with stent implantation into left anterior descending coronary artery was done within 6 hours after the onset of the chest pain. In both cases, the full restoration of the blood flow was achieved (TIMI flow III). Echocardiography showed the extensive antero-apicolateral area of akinesis with $\mathrm{EF}<40 \%$. Both patients were treated using standard medication (aspirin, clopidogrel, statin, $\beta$-blocker, and ACE inhibitor) during their hospitalization.

The transplantation of the progenitor cells was performed between days five and ten of AMI course (day 7 and
9 of the course of MI). The procedure was well tolerated with only mild angina at the time of the balloon inflation and the infusion of the progenitor cells. In both patients, the material for progenitor cell suspension was aspirated from sternum (three punctions, $35 \mathrm{~mL}$ of aspirate, four punctions, $45 \mathrm{~mL}$ of aspirate respectively). There were no procedural complications (no ventricular arrhythmia, thrombus formation, distal embolization, coronary artery occlusion or dissection). During hospitalization, no ventricular arrhythmia was documented. Troponin $\mathrm{T}$ and $\mathrm{CK}$ as a marker of myocardial necrosis were assessed before and after $24 \mathrm{~h}$, and one week after the cell transplantation. Both markers were decreasing during the time of observation. There were no significant changes in blood count.

Both patients completed three-month follow-up without any complications. Holter monitoring was performed during the ambulatory follow-up and did not reveal any significant arrhythmia.

The function of the left ventricle was assessed using echocardiography. Echocardiographic parameters showed improvement. Ejection fraction was increasing in both patients (from 35 to $40 \%$, resp. from 40 to $50 \%$ ).

\section{Discussion}

The capacity of adult bone marrow cells to convert into other tissues, referred as plasticity, is the background of their potential use in many fields of clinical medicine (8). Recent experimental studies revealed that bone marrow derived progenitor cells might contribute to the regeneration of ischemic myocardium and can enhance neovascularization (5).

The major goal of the intracoronary delivery of the autologous bone marrow cells is to improve the function of the left ventricle not only by the stimulation of neovascularization, but also by the enhancement of cardiac myocyte regeneration in the infarcted area $(6,10)$.

The data of TOPCARE-AMI Trial $(2,9)$ showed that intracoronary infusion of progenitor cells (either bonemarrow derived or circulating progenitor cells) is safe and feasible in patients after acute MI which has been treated using direct coronary artery stenting. This study also showed favourable effects on the left ventricle remodelling.

At this time, there is no possibility to postulate detailed conclusions for the cell therapy in acute MI. But our observation showed that this therapy was feasible and safe in both patients with acute MI. We did not observe any complications associated with the procedure. In both patients this treatment led to the increase of ejection fraction. Our observation also showed that the sternum can be used for aspiration of sufficient count of bone marrow cells.

In a view of the fact, that there are still many questions, which have to be answered, there is need of further investigation at this area. 


\section{Acknowledgments}

The study was supported by the research project MZO 00179906 and Cardiovascular Research Project of the Charles University Prague, No. MSM 0021620817 „Invasive approach to myocardial salvage and regeneration".

\section{References}

1. Beltrami AP, Barlucchi L, Torela D et al. Adult cardiac stem cells are multipotent and support myocardial regeneration. Cell 2003;114:763-76.

2. Britten MD, Abolmaali ND, Assmus B et al. Infarct remodeling after intracoronary progenitor cell treatment in patients with acute myocardial infarction (TOPCARE-AMI). Mechanistic insights from serial contrast enhanced magnetic resonance imaging. Circulation 2003;108:2212-8.

3. Fuchs S, Baffour R, Zhou Y et al. Transendocardial delivery of autologous bone marrow enhances collateral perfusion and regional function in pigs with chronic experimental ischemia. J Am Coll Cardiol 2001;37:1726-32.

4. Kawamoto A, Gwon $\mathrm{HC}$, Iwaguro $\mathrm{H}$ et al. Therapeutic potential of ex vivo expanded endothelial progenitor cells for myocardial ischemia. Circulation 2001;103:634-7.

5. Kocher AA, Schuster MD, Szabolcs MJ et al. Neovascularization of ischemic myocardium by human bone-marrow-derived angioblasts prevents cardiomyocyte apoptosis, reduces remodeling and improves cardiac function. Nature Med 2001;7:430-6.

6. Oh H, Bradfute SB, Gallardo TD et al. Cardiac progenitor cells from adult myocardium: Homing, differentiation, and fusion after infarction. Proc Natl Acad Sci USA $2003 ; 100: 12313-8$.
7. Orlic D, Kajstura J, Chimenti S et al. Mobilized bone marrow cells repair the infarcted heart, improving function and survival. Proc Natl Acad Sci USA2001; 98:10344-49.

8. Quesenberry PJ, Abedi M, Aliotta J et al. Stem cell plasticity: an overview. Blood Cells Mol Dis 2004;32:1-4.

9. Schachinger V, Assmus B, Britten $\mathrm{M}$ et al. Transplantion of progenitor cells and regeneration enhancement in acute myocardial infarction. Final one-year results of the TOPCARE-AMI Trial. J Am Coll Cardiol 2004;44:1690-99.

10. Tse HF, Kwong YL, Chan JFK, Lo G, Ho CL, Lau CP. Angiogenesis in ischemic myocardium by intramyocardial autologous bone marrow cell implantation. Lancet 2003;361:47-9.

Submitted February 2005.

Accepted May 2005.

Doc. MUDr. Radek Pudil, Ph.D.,

Charles University in Prague, Faculty of Medicine in Hradec Králové,

University Hospital Hradec Králové, 1st Department of Medicine, Sokolská 581,

50005 Hradec Králové, Czech Republic.

e-mail: pudilradek@yahoo.com 